\section{Relevância epidemiológica da \\ desnutrição e da obesidade em distintas classes sociais: métodos de estudo e aplicação à população brasileira}

\section{Relevance of under and overnutrition in different social classes: methods and application to the Brazilian population}

\section{Lenise Mondini}

Núcleo de Pesquisas Epidemiológicas em Nutrição e Saúde Universidade de São Paulo - NUPENS/USP

Av. Dr. Arnaldo, 715, 01246-904, São Paulo, SP - Brasil

\section{Carlos Augusto Monteiro}

Departamento de Nutrição Faculdade de Saúde Pública Universidade de São Paulo Coordenador Científico do NUPENS/USP

Av. Dr. Arnaldo, 715, 01246-904, São Paulo, SP - Brasil

\section{Resumo}

Buscou-se construir critérios de mesma especificidade no diagnóstico da desnutrição e da obesidade, de modo a produzir estimativas comparáveis para a prevalência desses eventos em distintos estratos socioeconômicos da população brasileira infantil e adulta. A fonte primária de dados é a Pesquisa Nacional sobre Saúde e Nutrição - PNSN, estudo do tipo transversal de base domiciliar, realizado no país em 1989. Foram considerados o conjunto das mulheres adultas estudadas pela PNSN, com idades entre 18 e 64 anos ( $n=15.669)$, o conjunto dos homens adultos com idades entre 20 e 64 anos $(n=14.235)$ e o conjunto das crianças com idade entre 6 e 35 meses ( $\mathrm{n}=3.641)$. Na avaliação do estado nutricional dos adultos empregou-se o Índice de Massa Corporal $\left(\mathrm{kg} / \mathrm{m}^{2}\right)$ e, na avaliação infantil, os índices peso/idade e peso/altura. Os percentis 5 e 95 da distribuição desses índices em uma população de referência foram utilizados como níveis críticos para o diagnóstico, respectivamente, da desnutrição e da obesidade. Os resultados obtidos evidenciam, entre outros aspectos, que a desnutrição infantil mostra-se controlada (prevalências muito baixas) mesmo em estratos da população com níveis muito modestos de renda familiar, enquanto a obesidade em mulheres adultas constitui problema de Saúde Pública (altas prevalências), mesmo para famílias situadas abaixo da linha da pobreza extrema. Tais evidências indicam a necessidade de se rever os modelos de causalidade tradicionalmente admitidos no país para a desnutrição e para a obesidade, ao mesmo tempo em que apontam a urgência de uma ampla revisão das prioridades e das estratégias de intervenção da Saúde Pública brasileira, no campo da nutrição.

Palavras-Chave: Desnutrição protéicocalórica. Obesidade. Classe social. Brasil. 


\section{Abstract}

The search for criteria of the same specificity in the diagnosis of both undernutrition and obesity is the main objective of this study. The selected criteria are applied to data collected by a nation-wide cross-sectional anthropometric survey undertaken in Brazil in 1989 as to produce comparable estimates for the prevalence of undernutrition and obesity in different socioeconomic strata. The study included 20-64 year-old male adults ( $\mathrm{n}=14,235), 18-64$ year-old female adults $(\mathrm{n}=15,669)$, and 6-35 month-old children ( $\mathrm{n}=3.641)$. Body Mass Index $(\mathrm{kg} /$ $\mathrm{m}^{2}$ ) was employed to assess nutritional status of adults and weight-for-age and weight-for-height indices were used for children. The $5^{\text {th }}$ and $95^{\text {th }}$ centiles of the distribution of these indices in a reference population were used as limits for the diagnosis of undernutrition and obesity, respectively. These results challenge the common belief that undernutrition is a relevant problem for the poor population, and that obesity only affects affluent strata. The need for an urgent revision of national public health nutrition priorities and interventions is stressed.

Keywords: Protein-energy malnutrition. Social class. Brazil.

\section{Introdução}

Decorrem do atendimento inadequado das necessidades energéticas do organismo dois dos principais distúrbios nutricionais que modernamente acometem os seres humanos: a desnutrição (atendimento inferior às necessidades) e a obesidade (atendimento superior às necessidades). Desnutrição e obesidade determinam consequências para a saúde dos indivíduos. A desnutrição condiciona crescimento e desenvolvimento deficientes, maior vulnerabilidade a doenças infecciosas, comprometimento de funções reprodutivas e redução da capacidade de trabalho ${ }^{1,2,3}$. A obesidade, por sua vez, está associada a várias doenças, entre as quais enfermidades cardiovasculares (e seus fatores de risco hipertensão e hiperlipidemias), diabetes mellitus e certos tipos de câncer ${ }^{4,5}$. Tanto a desnutrição como a obesidade são, portanto, agravos relevantes para a saúde dos indivíduos. A maior ou menor relevância epidemiológica destes distúrbios em uma dada sociedade - e, assim, a maior ou menor prioridade para o seu controle - dependerá essencialmente da magnitude que alcancem na população.

Com frequência assume-se que a desnutrição seja um problema relevante para os países não desenvolvidos, enquanto a obesidade o seria para os países desenvolvidos. Essa parece ser, entretanto, caracterização equivocada de realidade bastante mais complexa. Em primeiro lugar, países denominados desenvolvidos e não desenvolvidos estão longe de constituírem unidades homogêneas para a prevalência da desnutrição e da obesidade. Considere-se, por exemplo, a diferença que separa as elevadíssimas taxas de obesidade nos Estados Unidos daquelas observadas no Canadá ou na Inglaterra ${ }^{6}$, ou a maior magnitude da desnutrição nos países da África Sub-Sahariana diante dos países da América do Sul ${ }^{7}$. Em segundo lugar, países, sejam ou não desenvolvidos, tampouco são homogêneos, sendo antes constituídos por classes sociais cuja vulnerabilidade a distúrbios nutricionais é marcadamente 
distinta. Em terceiro lugar, internamente a cada uma das classes sociais, a magnitude da desnutrição e da obesidade poderá ser muito diferente para crianças e para adultos, para homens e para mulheres.

Um último e central aspecto a se considerar refere-se às tendências concomitantes de declínio da desnutrição e de ascensão da obesidade, observadas em sociedades em desenvolvimento que experimentam rápidas e intensas transformações em seu padrão de crescimento econômico e estrutura demográfica, fenômeno que caracteriza a denominada "transição nutricional",,9.

O conhecimento acerca da importância relativa da desnutrição e da obesidade em um dado país não prescinde, portanto, da comparação acurada da frequência destes distúrbios em diferentes segmentos e estratos de sua população. Tal aferição pressupõe a disponibilidade de inquéritos nutricionais com representatividade nacional e a existência de critérios comparáveis para a avaliação da frequência da desnutrição e da obesidade na população de crianças e de adultos. O desenvolvimento destes critérios e sua aplicação a diferentes estratos socioeconômicos da população brasileira constituem os objetivos deste artigo.

\section{Material e Métodos}

Os dados analisados neste estudo são provenientes da Pesquisa Nacional sobre Saúde e Nutrição - PNSN, um estudo do tipo transversal de base domiciliar, realizado no Brasil em 1989 pelo Instituto Brasileiro de Geografia e Estatística ${ }^{10}$. A amostra da PNSN, do tipo estratificada e probabilística, abrangeu 14.455 domicílios. Para efeito do presente estudo, foram considerados o conjunto das mulheres adultas estudadas pela PNSN com idades entre 18 e 64 anos $(\mathrm{n}=15.669)$, excluídas as gestantes, o conjunto dos homens adultos com idades entre $20 \mathrm{e}$ 64 anos ( $\mathrm{n}=14.235)$ e o conjunto de crianças com idade entre 6 e 35 meses $(n=3.641)$.

A escolha dos grupos etários mencionados se deveu essencialmente à maior facilidade de interpretação dos indicadores antropométricos de desnutrição e obesidade. A não inclusão de adultos com mais de 64 anos de idade decorre da dificuldade de se avaliar corretamente a altura de indivíduos idosos. A exclusão da população masculina entre 18 e 20 anos se deve ao ganho de peso que ainda ocorre nesta idade e que é atribuído ao final do processo de desenvolvimento, mais tardio no sexo masculino ${ }^{4}$.

Os critérios empregados para avaliação do estado nutricional das crianças e adultos estudados pela PNSN baseiam-se em índices antropométricos clássicos: a) peso relativo à idade (peso/idade) no diagnóstico da desnutrição infantil; b) peso relativo à altura (peso/altura) no diagnóstico da obesidade infantil; e c) Índice de Massa Corporal - IMC $\left(\mathrm{kg} / \mathrm{m}^{2}\right)$ no diagnóstico da desnutrição e da obesidade em adultos. Peso, altura e idade dos indivíduos foram obtidos na PNSN por pessoal contratado e treinado pelo IBGE. O peso foi obtido com auxílio de balança microeletrônica, estando os indivíduos sem sapatos e agasalhos. A altura foi medida na posição horizontal nas crianças com menos de dois anos de idade e na posição vertical, nas crianças mais velhas e adultos. A idade foi calculada a partir de registros de nascimento ou documentos equivalentes.

Estimativas acerca da prevalência da desnutrição infantil têm sido feitas, tradicionalmente, a partir da apuração da proporção de crianças com peso/idade abaixo de certos valores críticos pouco frequentes em populações gozando de boas condições de saúde e nutrição e que servem, assim, como população ou padrão de referência. Tais valores críticos usualmente correspondem ao percentil 3, 5 ou 10 da população de referência (ou seus equivalentes expressos em escore-z) ${ }^{11}$. A principal vantagem em se adotarem critérios baseados na distribuição percentilar das medidas de uma população de referência é a possibilidade de se conhecer previamente a especificidade com que se está fazendo o diagnóstico do déficit 
antropométrico (no caso dos percentis assinalados, especificidades de $97 \%$, 95\% e $90 \%$, respectivamente). As mesmas considerações se aplicam às estimativas relativas à prevalência da obesidade infantil, usualmente estabelecida a partir da proporção de crianças com peso/altura superior ao percentil 95 ou 97 de uma população de referência.

Estimativas sobre a prevalência da desnutrição em adultos têm sido feitas tradicionalmente a partir da proporção de indivíduos cujo Índice de Massa Corporal IMC (peso em kg dividido pela altura em metros ao quadrado) esteja abaixo de determinados valores críticos, usualmente $18,5 \mathrm{~kg} / \mathrm{m}^{2} \mathrm{ou}$, às vezes, $20,0 \mathrm{~kg} / \mathrm{m}^{2}$, independentemente da idade e sexo ${ }^{12,13}$. Estimativas sobre a prevalência da obesidade são feitas empregando-se como níveis críticos ora um valor fixo de IMC (habitualmente $25,0 \mathrm{~kg} / \mathrm{m}^{2}$ ou $30,0 \mathrm{~kg} / \mathrm{m}^{2}$ ), ora percentis do IMC segundo idade e sexo de uma população de referência (habitualmente o percentil 85 ou 95) ${ }^{12,14,15}$.

Objetivando dotar o diagnóstico da desnutrição e da obesidade da mesma especificidade - quer em crianças, quer em adultos - optou-se por estender aos adultos o "modelo normativo" de diagnóstico tradicionalmente aplicado às crianças. Mais especificamente, considerou-se como desnutrido todo adulto (ou criança) cujo IMC (ou peso/idade) encontre-se abaixo do percentil 5 de uma população de referência "livre de desnutrição"; como obeso IMC (ou considerou-se todo adulto (ou criança) cujo peso/altura) encontre-se acima do percentil 95 de uma população de referência "livre da obesidade".

Utilizou-se como população infantil de referência para os índices peso/idade e peso/altura o padrão NCHS/WHO (National Center of Health Statistics/World Health Organization $)^{16}$. O referido padrão, baseado em inquéritos representativos da população de crianças americanas, é amplamente utilizado em inquéritos antropométricos realizados em todo o mundo, aceitando-se que o mesmo represente satisfatoriamente o crescimento e as proporções corporais de crianças bem nutridas ${ }^{11}$.

Na ausência de um padrão de referência aceito universalmente para avaliação do IMC da população adulta, optou-se por tomar como população de referência o conjunto de indivíduos adultos estudados pela PNSN, com renda familiar de pelo menos 1,0 salário mínimo per capita. Níveis de renda próximos a este têm sido descritos como aqueles que permitiriam a satisfação das necessidades básicas das famílias brasileiras em alimentação, habitação e transporte ${ }^{17}$.

O quadro abaixo reproduz os valores de IMC correspondentes ao percentil 5 da população brasileira com renda de pelo menos um salário mínimo per capita, valores estes utilizados como valores críticos para o diagnóstico da desnutrição em adultos.

Valores críticos de IMC no diagnóstico da desnutrição em adultos

\begin{tabular}{ccc}
\hline Idade (anos) & Mulheres & Homens \\
\hline $18-19$ & 17,2 & - \\
$20-24$ & 17,6 & 18,4 \\
$25-29$ & 18,3 & 19,2 \\
$30-39$ & 19,3 & 19,6 \\
$40-49$ & 19,4 & 19,3 \\
$50-59$ & 20,0 & 20,0 \\
$60-64$ & 20,1 & 19,9 \\
\hline
\end{tabular}

Valores críticos únicos de IMC foram utilizados no caso do diagnóstico da obesidade em adultos: $28,4 \mathrm{~kg} / \mathrm{m}^{2}$ para a população masculina e $27,7 \mathrm{~kg} / \mathrm{m}^{2}$ para a população feminina. Tais valores correspondem ao percentil 95 da mesma população brasileira adulta de renda per capita de pelo menos 1,0 salário mínimo, restrita desta vez a indivíduos com menos de 25 anos de idade (18-24 anos no caso das mulheres e 20-25 anos no caso dos homens). A restrição a indivíduos jovens é condição necessária para assegurar que a população de referência utilizada seja efetivamente "livre da obesidade" e será apreciada em detalhe na discussão dos resultados deste artigo. 
Estimativas da prevalência de desnutrição e obesidade em crianças e em homens e mulheres são apresentadas para o país como um todo e para quatro estratos socioeconômicos da população brasileira definidos a partir da renda familiar mensal expressa em múltiplos de salários mínimos per capita (smpc): < 1/4 smpc; 1/4 -1/2 smpc; $1 / 2$-1,0 smpc; e $\geq 1,0$ smpc. Valores equivalentes ou próximos a 1/2 e a $1 / 4$ smpc foram usados no país na década de 80 para caracterizar, respectivamente, a linha da pobreza e a linha da pobreza absoluta ou da miséria ${ }^{18}$. A renda de 1,0 salário mínimo per capita, conforme já se mencionou, corresponde a valores compatíveis com a aquisição das necessidades básicas em alimentação, habitação e transporte de uma família brasileira ${ }^{17}$.

O controle da idade para o cálculo das estimativas da prevalência de desnutrição e obesidade em homens e mulheres, nos diferentes estratos econômicos, mostrou ser desnecessário, uma vez que a estrutura etária da população adulta é bastante semelhante nas diferentes faixas de renda.

A consistência e análise dos dados foram realizadas nos programas SPSS-Statistics Package for Social Sciences, versão 5.0 e EPIINFO, versão $6.04^{19,20}$.

\section{Resultados}

A Tabela 1 apresenta prevalências comparáveis de desnutrição e de obesidade para a população brasileira infantil e adulta. Nota-se que, entre crianças, a desnutrição tende a ser mais comum do que a obesidade (cerca de 1,5 vezes), não se notando diferenças entre os sexos. Entre adultos, inverte-se a situação, havendo predomínio da obesidade sobre a desnutrição, ligeiro no caso de homens ( 1,1 vezes) e acentuado

Tabela 1 - Prevalência (\%) de desnutrição e de obesidade segundo sexo na população de crianças e adultos. Brasil, 1989.

Table 1 - Prevalence (\%) of undernutrition and obesity by sex in children and adults (Brazil, 1989).

\begin{tabular}{lcccc}
\hline Sexo & \multicolumn{2}{c}{ Crianças } & Adultos & \\
& Desnutrição(1) & Obesidade(2) & Desnutrição(3) & Obesidade(4) \\
\hline Masculino & 13,4 & 8,5 & 8,3 & 9,5 \\
& $(11,1-15,7)$ & $(6,4-10,6)$ & $(7,5-9,1)$ & $(8,6-10,4)$ \\
Feminino & 12,9 & 9,6 & 7,6 & 20,0 \\
& $(10,6-15,2)$ & $(7,3-11,8)$ & $(6,9-8,2)$ & $(18,8-21,1)$ \\
\hline Total & 13,1 & 9,0 & 7,9 & 15,0 \\
& $(11,3-14,9)$ & $(7,4-10,6)$ & $(7,1-8,6)$ & $(13,6-16,4)$ \\
\hline
\end{tabular}

(1) Crianças de 6 a 35 meses com peso/idade < percentil 5 da população de referência.

(2) Crianças de 6 a 35 meses com peso/altura > percentil 95 da população de referência.

(3) Mulheres (18-64 anos) e homens (20-64 anos) com IMC < percentil 5 da população de referência.

(4) Mulheres (18-64 anos) e homens (20-64 anos) com IMC > percentil 95 da população de referência.

NOTA: A população de referência corresponde ao padrão internacional NCHS/OMS no caso das crianças, e à população brasileira de "alta renda" (considerados apenas os indivíduos jovens para efeito de cálculo do percentil 95), no caso dos adultos. O intervalo com $95 \%$ de confiança é fornecido entre parênteses.

(1) Children 6-35 months with weight-for-age $<5^{\text {th }}$ centile of the reference population.

(2) Children 6-35 months with weight-for-height $>95^{\text {th }}$ centile of the reference population.

(3) Women (18-64 year-old) and men (20-64 year-old) with $B M I<5^{\text {th }}$ centile of the reference population.

(4) Women (18-64 year-old) and men (20-64 year-old) with BMI $>95^{\text {th }}$ centile of the reference population.

NOTE: The reference population is the NCHS/WHO standard for children and the "high income" Brazilian population (only young individuals for the $95^{\text {th }}$ centile) for adults. Confidence intervals (95\%) in parenthesis.

no caso de mulheres (2,6 vezes). Considerando a magnitude absoluta dos eventos estudados, vê-se que o distúrbio nutricional que alcança maior frequência no país é a obesidade em mulheres adultas (prevalência de 20,0\%). Em situação intermediária encontra-se a desnutrição infantil (prevalência para os sexos combinados de 13,1\%). Ostentam menor frequência no país a desnutrição entre adultos de ambos os sexos, a obesidade em homens adultos e a obesidade infantil (prevalências ao redor de 8-9\%).

A Tabela 2 apresenta prevalências comparáveis de desnutrição e de obesidade para quatro estratos socioeconômicos da 
Tabela 2 - Prevalência (\%) de desnutrição e obesidade segundo renda familiar per capita na população de crianças e adultos. Brasil, 1989.

Table 2 - Prevalence (\%) of undernutrition and obesity by family income in children and adults (Brazil, 1989).

\begin{tabular}{lcccccc}
\hline $\begin{array}{l}\text { Renda familiar } \\
\text { (salários } \\
\text { mínimos } \\
\text { per capita) }\end{array}$ & \multicolumn{2}{c}{ Crianças } & \multicolumn{2}{c}{ Mulheres } & \multicolumn{2}{c}{ Homens } \\
\hline$<0,25$ & \multicolumn{7}{c}{ Desnutrição } & Obesidade & Desnutrição & Obesidade & Desnutrição & Obesidade \\
& 20,7 & 4,6 & 12,7 & 13,2 & 11,2 & 3,3 \\
$0,25-0,50$ & $(17,7-23,7)$ & $(2,8-6,5)$ & $(11,0-14,4)$ & $(11,6-14,8)$ & $(9,5-12,9)$ & $(2,2-4,4)$ \\
& 15,5 & 8,2 & 8,6 & 19,7 & 10,7 & 6,3 \\
$0,50-1,0$ & $(11,3-19,6)$ & $(5,5-11,0)$ & $(7,4-9,8)$ & $(17,9-21,5)$ & $(9,2-12,3)$ & $(4,9-7,7)$ \\
& 5,1 & 12,2 & 6,5 & 23,3 & 8,9 & 8,5 \\
$\geq 1,0$ & $(2,9-7,3)$ & $(8,0-16,4)$ & $(5,3-7,7)$ & $(21,0-25,5)$ & $(7,3-10,6)$ & $(7,1-10,2)$ \\
& 4,3 & 14,6 & 5,1 & 21,3 & 5,2 & 14,2 \\
& $(1,7-6,8)$ & $(10,6-18,5)$ & $(4,2-5,9)$ & $(19,4-23,2)$ & $(4,0-6,3)$ & $(12,6-15,8)$ \\
\hline
\end{tabular}

população brasileira, definidos a partir da renda familiar expressa em salários mínimos per capita.

\section{Renda familiar $<1 / 4$ smpc}

Deve-se dizer, inicialmente, que as famílias com renda per capita inferior a $1 / 4$ de salário mínimo, segundo estimativas ponderadas da PNSN, abrigavam $35,0 \%$ da população infantil do país (6-35 meses de idade), $16,6 \%$ da população adulta masculina (20-64 anos) e $17,6 \%$ da população adulta feminina (18-64 anos).

Nesse estrato de menor renda, como era de se esperar, a ocorrência de desnutrição excede a ocorrência de obesidade em quase cinco vezes, no caso das crianças, e em três vezes, no caso de homens adultos. $\mathrm{Na}$ população de mulheres adultas, entretanto, nota-se relativo equilíbrio entre os dois eventos, havendo, de fato, ligeiro predomínio da obesidade sobre a desnutrição. A consideração da magnitude absoluta alcançada pelos distúrbios nutricionais, nesse estrato de menor renda, torna claro que o principal problema das famílias que vivem abaixo da "linha da miséria” é a desnutrição infantil (20,7\%). Mulheres obesas $(13,2 \%)$, mulheres desnutridas (12,7\%) e homens desnutridos $(11,2 \%)$ apresentam-se como problemas de intermediária importância para esse estrato da população, enquanto a obesidade em crianças e em homens adultos não chega a alcançar expressão epidemiológica (prevalências aquém do valor crítico de 5\%).

\section{Renda familiar entre 1/4 e 1/2 smpc}

As famílias com renda per capita entre 1/4 e 1/2 salário mínimo abrigavam, à época da pesquisa, $24,3 \%$ da população infantil brasileira e $19,3 \%$ e $20,1 \%$, respectivamente, da população adulta masculina e feminina.

Nesse estrato de renda a ocorrência de desnutrição continua superior à ocorrência de obesidade nas crianças e em homens adultos, ainda que os diferenciais estejam substancialmente atenuados em relação ao estrato anterior de renda. $\mathrm{Na}$ população de mulheres adultas, a ocorrência da obesidade já excede em mais de duas vezes a ocorrência da desnutrição. A consideração da magnitude dos distúrbios nutricionais mostra que os maiores problemas enfrentados pela população pobre "não miserável" do país são a obesidade feminina $(19,7 \%)$ e a desnutrição infantil $(15,5 \%)$, nessa ordem. A desnutrição entre adultos perde importância nesse estrato da população (10,7\% e 8,6\% em homens e mulheres, respectivamente), enquanto a obesidade em crianças e em homens adultos já alcança expressão epidemiológica (8,2\% e $6,3 \%$, respectivamente). 
Renda familiar entre 1/2 e 1,0 smpc

As famílias com renda per capita entre 1/2 e 1,0 salário mínimo abrigavam, à época da pesquisa, $20,2 \%$ da população infantil brasileira e $25,3 \%$ e $24,2 \%$, respectivamente, da população de homens e mulheres adultos.

Nesse estrato da população, cuja renda ainda não seria suficiente para suprir o conjunto das necessidades básicas das famílias, a ocorrência de desnutrição é equivalente à ocorrência de obesidade apenas na população de homens adultos. Na população infantil e na população de mulheres adultas, a ocorrência de obesidade excede em mais de duas e em mais de três vezes, respectivamente, a ocorrência de desnutrição. A consideração da magnitude absoluta dos distúrbios nutricionais indica que a obesidade em mulheres adultas (23,3\%) é o principal problema nesse estrato de renda da população, seguido, com certa distância, pela obesidade infantil (12,2\%). A desnutrição em crianças e em adultos continua perdendo importância, sendo que no caso das crianças o problema virtualmente já não mais alcança expressão epidemiológica $(5,1 \%)$.

\section{Renda familiar $\geq 1,0$ smpc}

As famílias com renda per capita de pelo menos 1,0 salário mínimo abrigavam, à época da pesquisa, $20,5 \%$ da população infantil brasileira e $38,8 \%$ e $38,2 \%$, respectivamente, da população de homens e mulheres adultos.

Nesse estrato de maior renda familiar, como era de se esperar, o risco de obesidade predomina largamente sobre o risco de desnutrição, seja na população infantil, seja na população adulta. A consideração da magnitude absoluta dos distúrbios nutricionais indica que o maior problema enfrentado por esse grupo da população é a obesidade feminina (21,3\%), seguida pela obesidade em crianças e em homens adultos (14,6\% e 14,2\%, respectiva-mente). A desnutrição não alcança expressão epidemiológica nesse estrato, seja com relação às crianças, seja com relação à população adulta.

\section{Discussão}

Dois aspectos do presente trabalho merecem ser considerados com maior detalhe: o primeiro, de ordem metodológica, inclui considerações sobre os índices antropométricos e a adequação dos padrões de referência utilizados no diagnóstico da desnutrição e da obesidade; o segundo refere-se às implicações para a Saúde Pública que decorrem do conjunto de nossos achados.

\section{Índices antropométricos e padrões de referência}

Em relação à população infantil, os índices antropométricos utilizados neste estudo são aqueles classicamente adotados na avaliação do estado nutricional de crianças: peso relativo à idade no diagnóstico de desnutrição e peso relativo à altura no diagnóstico da obesidade. O peso baixo para a idade reflete tanto déficits de crescimento (altura para idade) quanto o baixo peso para a altura. Estudos prospectivos têm emprestado significado biológico ao índice peso/idade na medida em que evidenciam estreita associação entre este indicador e o risco de mortalidade na infância ${ }^{1}$. O peso relativo à altura, em tese, reflete variações tanto no tecido adiposo quanto na massa magra dos indivíduos. Entretanto, em crianças de pequena idade, índices peso/altura elevados geralmente não se devem ao aumento da massa muscular, mas sim à obesidade.

Em relação à população adulta, há que se distinguir o diagnóstico da desnutrição e da obesidade. No caso do diagnóstico da desnutrição, o emprego da relação peso/ altura, expressa por meio do Índice de Massa Corporal - IMC, encontra sustentação no fato de que ambos os compartimentos da massa corporal - o tecido adiposo e a massa magra - são 
afetados pela desnutrição. Ademais, a relação entre baixos valores de IMC e maior ocorrência de morbi-mortalidade e alterações da função reprodutiva tem sido demonstrada em estudos empíricos ${ }^{21,22,23,24}$. O fato de que valores elevados de IMC, em tese, não façam distinção entre acúmulo de tecido adiposo (obesidade) e aumentos na massa magra, torna menos seguro o emprego desse índice no diagnóstico da obesidade adulta. Ainda assim, recomendase o seu uso diante das dificuldades operacionais relacionadas ao emprego de medidas diretas da composição corporal, como a medida de bioimpedância ou a medida de dobras cutâneas ${ }^{4}$. Importa também notar que são geralmente altas as correlações encontradas entre o IMC e a porcentagem de gordura aferida por medidas diretas da composição corporal ${ }^{25,26}$.

Uma outra limitação do IMC, talvez menos importante, refere-se à sua relação com a altura dos indivíduos. Normalmente se assume que o IMC é independente da altura, muito embora esta condição nem sempre seja verificada, principalmente entre os mais jovens ${ }^{27}$. Na população estudada, no entanto, verificou-se que IMC e peso são altamente correlacionados, enquanto que IMC ( $\left.\mathbf{k g} / \mathbf{m}^{2}\right)$

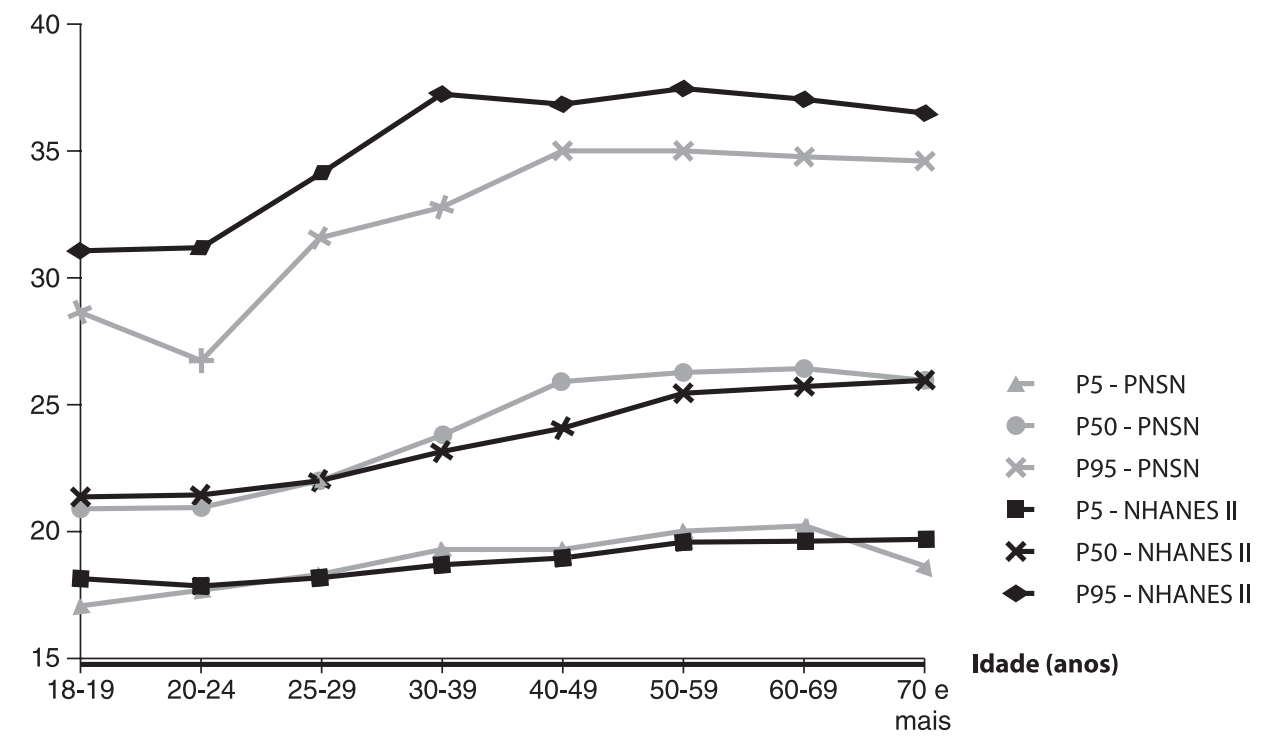

Figura 1 - Percentis do IMC de mulheres adultas segundo faixa etária: população brasileira de "alta renda" (PNSN) versus população americana (NHANES)

Figure 1 - Centiles of BMI in adult womem by age:" "high income"Brazilians (PNSN) versus U.S. population (NHANES) os coeficientes de correlação entre IMC e altura apresentam magnitudes muito baixas para homens e mulheres de diferentes faixas etárias (entre -0,03 e 0,08) ${ }^{28}$.

Com relação aos padrões antropométricos de referência, pouco se tem a comentar no caso da avaliação do estado nutricional infantil. Conforme mencionado anteriormente, o padrão NCHS/WHO apresenta a vantagem de ser universalmente utilizado e de representar satisfatoriamente o potencial de crescimento físico de populações infantis bem nutridas, condição já demonstrada paraa população brasileira em estudo anterior dos autores ${ }^{29}$.

No caso da avaliação do IMC da população adulta, ao contrário, a escolha do padrão de referência requereu investigação mais detalhada. De início, considerou-se a possibilidade de utilizar como padrão de referência a distribuição do IMC de adultos, observada na mesma população americana que deu origem ao padrão NCHS/WHO para avaliação infantil e disponibilizada a partir da realização da pesquisa NHANES II (Second National Health and Nutrition Examination Survey) ${ }^{30}$. O padrão NHANES II garantiria a condição de se trabalhar com a distribuição de 


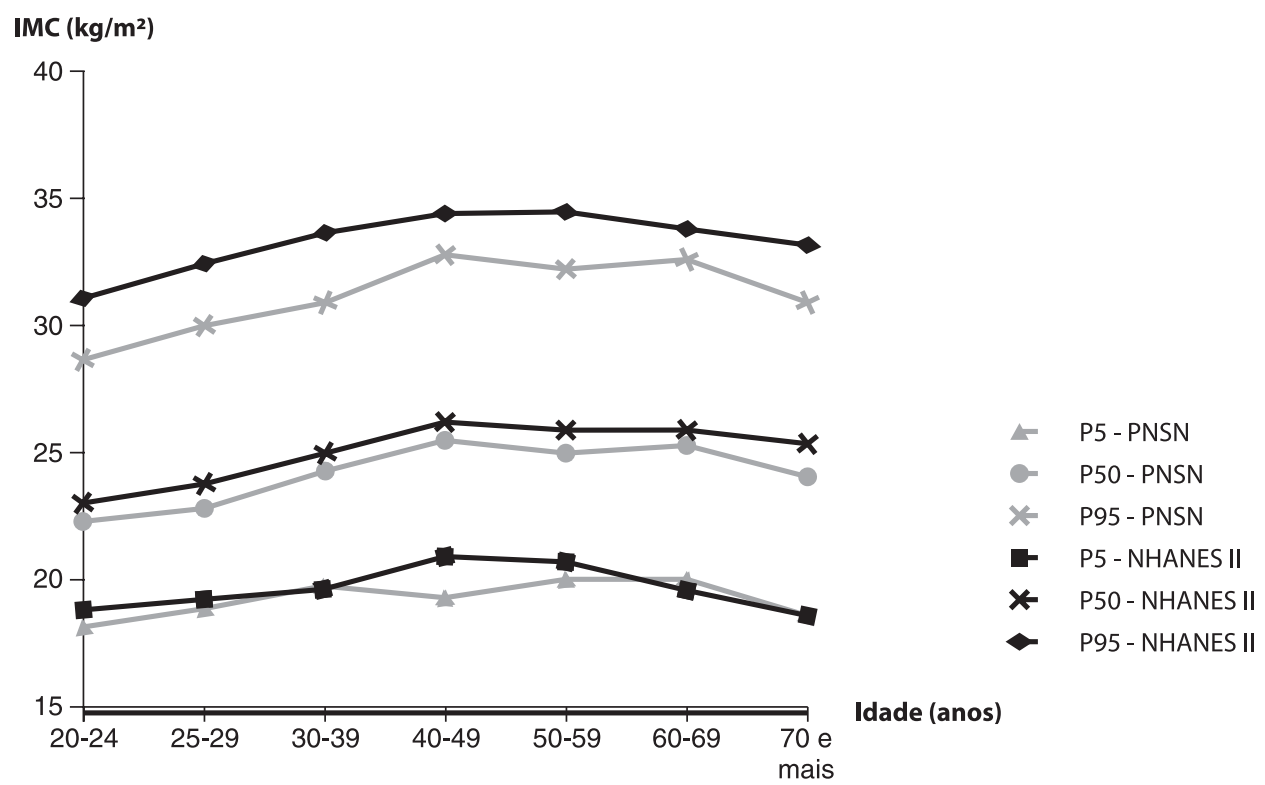

Figura 2 - Percentis do IMC de homens adultos segundo faixa etária: população brasileira de "alta renda" (PNSN) versus população americana (NHANES)

Figure 2 - Centiles of BMI in adult mem by age: "high income" Brazilians (PNSN) versus U.S. population (NHANES)

medidas de uma população "livre de desnutrição”. Entretanto, estudos sobre a composição corporal da população americana indicam proporções consideráveis de obesos ${ }^{14}$. Assim, passouse a cogitar como alternativa a distribuição do IMC da população brasileira adulta de "alta renda" examinada pela PNSN.

A Figura 1 compara a distribuição do IMC de mulheres americanas (NHANES II) e de mulheres brasileiras com renda familiar de pelo menos 1,0 salário mínimo per capita, destacando o comportamento dos percentis 5, 50 e 95 ao longo das idades. A Figura 2 procede à mesma comparação com relação à população masculina.

Nota-se, inicialmente, grande semelhança com relação ao comportamento dos valores de IMC correspondentes ao percentil 5 das duas populações, valendo tal afirmação para ambos os sexos. Essa situação indica que os resultados sobre a prevalência da desnutrição em adultos, obtidas por este estudo, pouco seriam modificados caso se utilizasse a população americana como padrão de referência.

A inspeção dos valores correspondentes ao percentil 95 do IMC da população feminina e masculina americana confirma a não recomendação dessa população como padrão de referência para avaliação da ocorrência de obesidade em adultos. Em todas as idades, para homens e para mulheres, aquele valor ultrapassa $30,0 \mathrm{~kg} /$ $\mathrm{m}^{2}$, nível de IMC que estudos de composição corporal associam claramente à obesidade $^{15,31}$. Situação semelhante percentis 95 acima de $30,0 \mathrm{~kg} / \mathrm{m}^{2}$ - é detectada para homens e mulheres brasileiros de alta renda a partir da idade de 25 anos, porém não antes desta idade. Em face dessa situação optou-se por utilizar como população de referência a população brasileira de adultos de alta renda, restringindo-a, no caso do diagnóstico da obesidade, e só neste caso, à população com idade inferior a 25 anos (18 a 24 anos para mulheres e 20 a 24 anos para homens).

\section{Implicações para a Saúde Pública}

Os resultados encontrados por este estudo revelam, de modo contundente, quão imprecisa é a afirmação de que a desnutrição é um problema de toda população pobre do país, enquanto a 
obesidade apenas o seria para os mais ricos. Destaca-se, inicialmente, o fato auspicioso revelado pela virtual ausência da desnutrição infantil já no estrato de renda entre $1 / 2$ e 1,0 salário mínimo per capita, estrato que reúne famílias cujo poder aquisitivo, ainda muito modesto, seria teoricamente insuficiente mesmo para atender às necessidades básicas das famílias ${ }^{17}$. Considerando o contingente de crianças pertencente a esse estrato de renda e o contingente pertencente ao estrato de renda imediatamente superior, também protegido, constata-se que para mais de $40 \%$ da população infantil do país a desnutrição já não mais representaria um problema de grande relevância epidemiológica.

Igualmente notável, mas evidentemente nada auspicioso, é constatar que a obesidade alcança prevalências elevadas, mesmo nos estratos familiares de menor renda. Situação destacada é a da população adulta feminina que está exposta a taxas elevadas de obesidade, mesmo quando a renda familiar encontra-se abaixo da linha da pobreza absoluta (renda mensal per capita de menos de um quarto de salário mínimo).

Tanto o fato de a desnutrição infantil se mostrar relativamente controlada em estratos da população com níveis ainda muito modestos de renda, quanto a evidência de a obesidade feminina não poupar mesmo as famílias mais pobres do país, sugerem a necessidade de se rever os modelos de causalidade tradicional-mente admitidos no país para os distúrbios da nutrição. No caso da desnutrição infantil será importante examinar a importância do poder aquisitivo das famílias "vis-à-vis" outros determinantes, como o acesso das mesmas a serviços públicos, a qualidade do cuidado infantil, entre outros ${ }^{32,33}$. No caso da obesidade feminina, os achados do presente estudo, somados a evidências de outros estudos que examinam tendências temporais da obesidade no país ${ }^{34}$, indicam que a educação, o nível de informação da população sobre os riscos associados ao ganho de peso na idade adulta e fatores culturais e comportamentais tendema assumir papel de destaque na epidemiologia da obesidade, a exemplo do que já ocorre nos países desenvolvidos ${ }^{31}$.

As modificações implícitas na causalidade da desnutrição e da obesidade no país e a surpreendente escala de magnitude alcançada por estes distúrbios, nos diferentes estratos de sua população, apontam a necessidade urgente de uma ampla revisão das prioridades e das estratégias de intervenção da Saúde Pública brasileira, no campo da nutrição.

\section{Summary}

The search for criteria of the same specificity in the diagnosis of both undernutrition and obesity is the main objective of this study. The selected criteria are applied to data collected by a nationwide cross-sectional anthropometric survey undertaken in Brazil in 1989 as to produce comparable estimates for the prevalence of undernutrition and obesity in different socioeconomic strata. The study included 20 64 year-old male adults ( $n=14,235), 18-64$ year-old female adults $(n=15,669)$ and $6-35$ month-old children ( $n=3.641)$. Body Mass Index $\left(\mathrm{kg} / \mathrm{m}^{2}\right)$ was employed to assess nutritional status of adults and weight-forage and weight-for-height indices were used for children. The $5^{\text {th }}$ and $95^{\text {th }}$ centiles of the distribution of these indices in a reference population were used as limits for the diagnosis of undernutrition and obesity, respectively. Comparable prevalences of undernutrition and obesity were observed in four socioeconomic strata. In extremely poor families, undernutrition exceeds obesity among children and adult males, about five and three times respectively; in the same group,obese women slightly exceed undernourished women. In poor families, undernutrition still exceeds obesity among children and adult males, and obesity exceeds undernutrition nearly two-fold among adult females. In middle income families undernutrition is equivalent to obesity among adult males, and obesity far exceeds undernutrition among children and adult females. In high income families, obesity predominates among both infant and adult population. Two relevant 
findings emerge from our analysis of PNSN: prevalence of infant undernutrition is relatively low even among families with modest levels of income, and prevalence of women's obesity is high even among extremely poor families. These results challenge the common belief that undernutrition is a relevant problem for the poor population, andthat obesity only affects affluent strata. The need for an urgent revision of national public health nutrition priorities and interventions is stressed.

\section{Referências}

1. Pelletier DL. The relationship between child anthropometry and mortality in developing countries: implications for policy and future research. Ithaca (NY): Cornell University; 1991. (Monograph 13).

2. Administrative Committee on Coordination/ Subcommittee on Nutrition. Some options for improving nutrition in the 1990s. SCN News 1991;(7 Suppl.).

3. Ferro-Luzzi A, Branca F, Pastore G. Body mass index defines the risk of seasonal energy stress in the Third World. Eur J Clin Nutr 1994; 48 Suppl. 3:S16578.

4. World Health Organization. Physical status: the use and interpretation of anthropometry. Geneva; 1995. (WHO - Technical Report Series, 854).

5. Pi-Sunyer FX. Health implications of obesity. Am J Clin Nutr. 1991; 53: 1595S-603S.

6. Millar WJ, Stephens T. The prevalence of overweight and obesity in Britain, Canada and United States. Am J Public Health 1987; 77: 38-41.

7. De Onís M, Monteiro CA, Akré J, Clugston G. The worldwide magnitude of protein-energy malnutrition: an overview from the WHO Global Database on Child Growth. Bull World Health Organ 1993; 71: 703-12.

8. Popkin BM. Nutrition patterns and transitions. Popul Dev Rev 1993; 19: 138-57.

9. Popkin BM. The nutrition transition in low income countries: an emergency crisis. Nutr Rev 1994; 52:285-98.

10. Fundação IBGE/Instituto de Pesquisa Econômica Aplicada/Instituto Nacional de Alimentação e Nutrição. Pesquisa nacional sobre saúde e nutrição: manual do entrevistador - questionário de saúde e nutrição. Brasília; 1988. (PNSN 3.02).

11. WHO Working Group. Use and interpretation of anthropometric indicators of nutritional status. Bull World Health Organ 1986; 64: 929-41.

12. Waaler HT. Height, weight and mortality the Norwegian experience. Acta Med Scand 1984; 215 Suppl. 679: 1-56.

13. Ferro-Luzzi A, Sette S, Franklin M, James WPT. A simplified approach of assessing adult chronic energy deficiency. Eur J Clin Nutr 1992; 46: 173-86.

14. Abraham S, Carroll MD, Najjar MF, Fulwood R. Obese and overweigth adults in the United States. Vital Health Stat Ser 1983; 11 (230): 1-93.

15. Bray AG. Obesity: basic considerations and clinical approaches. Dis Mon 1989; 18: 449-540.

16. Hamill PVV, Drizd TA, Johnson CL, Reed RB, Roche AF, Moore WMM. Physical growth: National Center of Health Statistics percentiles. Am J Clin Nutr 1979; 32: 607-29.

17. DIEESE. Departamento Intersindical de Estatística e Estudos Sócio-Econômicos. Boletim 1990 Jan.;19.

18. Jaguaribe H, Silva NV, Abreu MP, Ávila FB, Fritsch W. Brasil: reforma ou caos. Rio de Janeiro: Paz e Terra; 1989.

19. Norusis MJ. Advanced statistics SPSS/PC+ for the IBM PC/XT/AT [computer program]. Version 5.0. Chicago: SPSS Inc.; 1992.

20. Centers for Disease Control and Prevention/World Health Organization. Epidemiology Program Office Atlanta - Epi-Info [computer program] Version 6.04. Georgia (USA); 1996.

21. Naidu AN, Rao NP. Body mass index: a measure of nutritional status in Indian populations. Eur J Clin Nutr 1994; 48 Suppl. 3: S131-40.

22. Garcia M, Kennedy E. Assessing linkages between body mass index and morbidity in adults: evidences from four developing countries. Eur J Clin Nutr 1994; 48 Suppl. 3: S90-7.

23. Campbell P, Ulijaszek SJ. Relationships between anthropometry and retrospective morbidity in poor men in Calcuta, Índia. Eur J Clin Nutr 1994;48:507-12.

24. Kusin JA, Kardjat S, Renqvist U, Goei K. Reprodution and maternal nutrition in Madura, Indonesia. Trop Geogr Med. 1992; 44: 248-55.

25. Bray AG. Obesidad. In: Organización Panamericana de la Salud. Conocimientos actuales sobre nutrición. Washington (DC); 1991. p. 28-46-(OPAS Publicación Científica, 532).

26. Hortobágyi T, Israel RG, O’Brien KF. Sensitivity and specificity of the Quetelet index assess obesity in men and women. Eur J Clin Nutr 1994; 48: 369-75.

27. Garn S.M, Leonard WR, Hawthorne VM. Three 
limitations of body mass index. Am J Clin Nutr 1986; 44: 996-7.

28. Mondini L. Desnutrição e obesidade no Brasil: relevância epidemiológica e padrões de distribuição intra-familiar em diferentes estratos econômicos e regionais. São Paulo; 1996. [Tese de Doutorado Faculdade de Saúde Pública da USP].

29. Monteiro CA, Benício MHD’A, Gouveia NC. Saúde e nutrição das crianças brasileiras no final da década de 80. In: IBGE/UNICEF. Perfil estatístico de crianças e mães no Brasil: aspectos de saúde e nutrição de crianças no Brasil-1989. Rio de Janeiro; 1992. p. $19-42$.

30. Najjar MF, Rowland M. Anthropometric reference data and prevalence of overweight United States,
1976-80. Vital Health Stat., Ser.11 1987; (238): 1-73.

31. World Health Organization. Diet, nutrition and the prevention of chronic diseases. Geneva; 1990. (WHO - Technical Report Series, 797).

32. Mason J. Assessing, analyzing and monitoring nutrition situations. Geneva: Administrative Committee on Cordination/Subcommittee on Nutrition; 1992. p. 16-29.

33. Longhurst R, Tomkins A. The role of care in nutrition-a neglected essential ingredient. SCN News 1995;12:1-5.

34. Monteiro CA, Mondini L, Souza ALM, Popkin B.M. The nutrition transition in Brazil. Eur J Clin Nutr 1995; 49: 105-13. 